\title{
Non-Integral Technique and Differential Transformation Method for MHD Boundary Layer Flow of an Incompressible Fluid Past A Flat Plate
}

\author{
B. B. Singh ${ }^{1}$ and I. M. Chandarki $\mathbf{i}^{1,2}$ \\ ${ }^{1}$ Department of Mathematics, \\ Dr. Babasaheb Ambedkar Technological University, Lonere-402103 Raigad(M.S.)India \\ Email: brijbhansingh@yahoo.com \\ ${ }^{2}$ Department of General Science and Engg., \\ N. B. Navale Sinhgad College of Engineering, Solapur-413255 (M.S.) \\ Email: imran_2204@ yahoo.co.in
}

\begin{abstract}
This paper deals with a Non-Integral Technique (NIT) and Differential Transformation Method (DTM) which are sufficiently accurate and efficient methods for approximate solutions of the MHD boundary layer flow of an incompressible fluid past a flat plate for a wide range of the magnetic parameter. The proposed solutions are first obtained by NIT by utilizing a known solution of another differential equation and then by DTM, where a group of transformations are used to reduce the boundary value problem into a pair of initial value problems, which are then solved by means of the differential transformation method. The proposed method yields closed series solutions of the boundary layer equations, which can then be calculated numerically. The pertinent parameter appearing in the problem is discussed graphically and presented in table. From the numerical values, it is evident that as the value of the magnetic parameter increases, the skin-friction on the surface of the plate also increases, and it becomes unity when the magnetic parameter becomes infinity. This is indicative of the fact that even if the magnetic parameter is infinitely large, the skin-friction will be finite only.
\end{abstract}

Keywords: Differential Transformation Method, Hydromagnetic boundary layer, local error, Non-Integral Technique, skin-friction. 


\section{Introduction}

The simplest example of the application of the boundary layer equation is afforded by the flow along a very thin plate. Historically, this is the first example illustrating the application of Prandtl's boundary layer theory ; it was discussed by Blasius [4] in his doctor's thesis. Subsequently, problems like these were also solved by Prandtl [13], Bairstow [3], Goldstein [9], Toepfer [17], Singh and Chandarki [15], etc.

The first objective of the present paper is to find out the approximate solutions of the MHD boundary layer equation governing the flow of an incompressible fluid past a flat plate by utilizing a known solution of another equation on the lines of Dey [6] who found out the approximate solutions of the Falkner-Skan equation [8].

Secondly the study employs the differential transformation method to obtain power series solutions of the same boundary-layer problem. A group of transformations are used to reduce the third-order nonlinear boundary value problems to a pair of initial value problems. These problems are then solved by the differential transformation method. The study concludes by comparing the current numerical results with those given by other integral approximation methods and Non Integral Technique considered in first part in order to verify the accuracy of the methods.

\section{Formulation of the Problem}

Let us consider a steady two-dimensional flow of a viscous fluid on a flat plate in the presence of a transverse magnetic field strength $B_{y}(x)$. In the case of a small electric conductivity and large transverse magnetic field $B_{y}(x)$, the boundary layer equations are given by

$$
\begin{gathered}
\frac{\partial u}{\partial x}+\frac{\partial v}{\partial y}=0 \\
u \frac{\partial u}{\partial x}+v \frac{\partial u}{\partial y}=U \frac{d U}{d y}+\nu \frac{\partial^{2} u}{\partial y^{2}}-\frac{\sigma B_{y}^{2}}{\rho}\left(u-U_{\infty}\right)
\end{gathered}
$$

subject to the boundary conditions

$$
\left\{\begin{array}{l}
u=0, \quad v=0, \quad \text { at } y=0 \\
u(\infty)=U_{\infty} \text { at } y=\infty
\end{array}\right.
$$

where $y=\infty$ denotes the edge of the boundary layer and $U_{\infty}$ is a constant potential flow velocity.

In the above equation, $x$ is the co-ordinate measured along the surface starting 
from the slit location in the direction of the motion ; $y$ is the co-ordinate normal to the surface; $u, v$ are the velocity components in the directions of $x-$ and $y-$ axes respectively ; $g$ is the acceleration due to gravity; $\rho$ is the fluid density; $\sigma$ is the electrical conductivity and $\nu$ is the kinematic viscosity.

Let $\psi(x, y)$ denote the stream-function such that

$$
u=\frac{\partial \psi}{\partial y}, \quad v=-\frac{\partial \psi}{\partial x}
$$

which satisfies the equation of continuity (1). The substitution of (4) in (2) leads to the following differential equation

$$
\frac{\partial \psi}{\partial y} \frac{\partial^{2} \psi}{\partial x \partial y}-\frac{\partial \psi}{\partial x} \frac{\partial^{2} \psi}{\partial y^{2}}=\nu \frac{\partial^{3} \psi}{\partial y^{3}}-S(x)\left(\frac{\partial \psi}{\partial y}-U_{\infty}\right)
$$

where $S(x)=\frac{\sigma B_{y}^{2}}{\rho}$.

As the flow is taking place past a flat plate, $U(x)=U_{0}=$ constant.

As a result, the term $U \frac{d U}{d x}$ vanishes.

Now let us set

$$
S(x)=S_{0} x^{\epsilon}
$$

and put $\eta=\left(\frac{U_{\infty}}{2 \nu x}\right)^{1 / 2}, \psi=\left(2 \nu U_{\infty} x\right)^{1 / 2} f(\eta)$ to study the similarity solution of (2).

The condition that each term in the equation contains the same degree in $x$ gives $\epsilon=-1$, and the equations (4) - (5) reduce to

$$
f^{\prime \prime \prime}+f f^{\prime \prime}+\beta\left(1-f^{\prime}\right)=0
$$

accompanied by the boundary conditions

$$
f(0)=f^{\prime}(0)=0, \quad f^{\prime}(\infty)=1
$$

where $\beta=\frac{S_{0}}{U-\infty} \geq 0$ is the magnetic parameter.

\section{Mathematical Analysis}

\subsection{Non- Integral Technique(NIT):}

The genesis of the present method is to solve (6) using a known solution of another third order non-linear equation having the same boundary conditions as those of (6). Briefly, the variables in (6) are scaled with the known solution using a parameter $\alpha$. The square of the error introduced as a result of the scaled variables introduced in (6) is minimized to obtain $\alpha$. 
The present choice of a differential equation, having the same boundary conditions as those of (6), in fact should provide a guidance in the selection of the trial functions for approximate methods proposed by many investigators. On the other hand, a trial function that itself is a solution of another equation having the same boundary conditions should be ideal. In this way, one can ensure that the nature of the highest order derivative in (6)is preserved, and the trial function satisfies the same boundary conditions as the dependant variable in (6).

For the present analysis, a known solution of a differential equation having the same boundary conditions as these of (6) is readily available if we consider the equation as

$$
\frac{d^{3} g}{d \eta^{3}}-\frac{d g}{d \eta}+1=0
$$

subject to the boundary conditions

$$
g=\frac{d g}{d \eta}=0, \quad \text { at } \quad \eta=0 \quad \frac{d g}{d \eta} \rightarrow 1 \quad \text { as } \quad \eta \rightarrow \infty
$$

Here it may be noted that (8) is a particular case of (6) for $\beta \rightarrow \infty$, and can be obtained from (6) by using the transformations

$$
y=\beta^{1 / 2} \eta, \quad g=\beta^{1 / 2} f
$$

and letting $\beta \rightarrow \infty$.

The closed form solution of (8) is

$$
\frac{d g}{d \eta}=1-e^{-\eta}
$$

Although (6) and (8) are the two different non-linear equations, they are of the same order, and their boundary conditions are similar. In order to find the approximate solutions of (6)-(7) on the lines of Dey [6], let us consider the following transformations:

$$
\left\{\begin{array}{l}
f(\eta)=\frac{g(\alpha \eta)}{\alpha}, \quad \frac{d f(\eta)}{d \eta}=\frac{d g(\alpha \eta)}{d \eta}, \quad \frac{d^{2} f(\eta)}{d \eta^{2}}=\alpha \frac{d^{2} g(\alpha \eta)}{d \eta^{2}} \\
\frac{d^{3} f(\eta)}{d \eta^{3}}=\alpha^{2} \frac{d^{3} g(\alpha \eta)}{d \eta^{3}}
\end{array}\right.
$$

Using (12) in (6), one can obtain

$$
\alpha^{2} g^{\prime \prime \prime}+g g^{\prime \prime}+\beta\left(1-g^{\prime}\right)=e(\alpha, \eta)
$$

where $e(\alpha, \eta)$ is the local error introduced due to the introduction of (12). Henceforth, the prime denotes the differentiation with respect to $\eta$. 
The following conditions for (13) are the same as given in (9).

Following Annamalai et al. [1], squaring the error we estimate $E$, given by

$$
E=\int_{0}^{\infty} e^{2}(\alpha, \eta) d \eta
$$

and then minimize; i.e., by setting

$$
\frac{d E}{d \alpha}=0
$$

The final expression for $\alpha$ that follows from (11), (13) -(15) leads as

$$
\alpha^{2}=\beta+0.2205
$$

This relation is valid for small values of $\beta$. Using the equation for large $\beta$, as is clear from Evans [7], obtained from (6) and the transformations in (10), it can be shown for large $\beta$ that

$$
\alpha^{2}=1+\frac{0.2205}{\beta}
$$

Once $\alpha$ is known, the approximate values of the non-dimensional skin-friction $C_{f}$ can be estimated from the quantity

$$
C_{f}=\alpha g^{\prime \prime}(0), \quad g^{\prime \prime}(0)=1
$$

\subsection{Differential Transformation Method:}

In order to find the series solution of equation (6) for a family of values of magnetic parameter $\beta$, it is first necessary to define a dependent variable $G(\eta)$, i.e.,

$$
G(\eta)=\frac{\partial f(\eta)}{\partial \beta}
$$

Differentiating (6)-(7) with respect to $\beta$ gives

$$
\frac{d^{3} G(\eta)}{d \eta^{3}}+f(\eta) \frac{d^{2} G(\eta)}{d \eta^{2}}+G(\eta) \frac{d^{2} f(\eta)}{d \eta^{2}}+\left(1-\frac{d f(\eta)}{d \eta}\right)-\beta \frac{d G(\eta)}{d \eta}=0 .
$$

The boundary conditions are given by

$$
G(0)=\frac{d G(0)}{d \eta}=0, \quad \frac{d G(\infty)}{d \eta}=0
$$


The method of superposition is used together with a group of transformations to solve the boundary-layer equation given in (2). Initially, the following expression is defined:

$$
G(\eta)=P(\eta)+C Q(\eta)
$$

where $C$ is a constant to be determined.

Substituting (22) into (20) gives the following pair of initial value problems:

$$
\frac{d^{3} P(\eta)}{d \eta^{3}}+f(\eta) \frac{d^{2} P(\eta)}{d \eta^{2}}+P(\eta) \frac{d^{2} f(\eta)}{d \eta^{2}}-\beta \frac{d P(\eta)}{d \eta}=\frac{d f(\eta)}{d \eta}-1
$$

with the initial conditions of

$$
P(0)=\frac{d P(0)}{d \eta}=\frac{d^{2} P(0)}{d \eta^{2}}=0,
$$

and

$$
\frac{d^{3} Q(\eta)}{d \eta^{3}}+f(\eta) \frac{d^{2} Q(\eta)}{d \eta^{2}}+Q(\eta) \frac{d^{2} f(\eta)}{d \eta^{2}}-\beta \frac{d Q(\eta)}{d \eta}=0
$$

with the initial conditions of

$$
Q(0)=\frac{d Q(0)}{d \eta}=0, \quad \frac{d^{2} Q(0)}{d \eta^{2}}=1 .
$$

Substituting the boundary condition at infinity from (21) into (22) gives the value of the parameter $C$ as

$$
C=-\frac{\frac{d P(\infty)}{d \eta}}{\frac{d Q(\infty)}{d \eta}} .
$$

To solve (6) at $\beta=\Delta \beta$; (6) is first solved for the case of $\beta=0$ in order to establish the function $f(\eta)$ and its derivatives which appear in equations (23) and (25). Solving equations (23)-(26) then gives $P(\eta)$ and $Q(\eta)$, and their derivatives. The value of $C$ is obtained by substituting $\frac{d P(\infty)}{d \eta}$ and $\frac{d Q(\infty)}{d \eta}$ in to equation (27). Given $C$, the values of $G(\eta)$ are derived form (22) and are then substituted into the rearranged from of (19) given below to give the solutions of $f(\eta)$ at $\beta=\Delta \beta$, i.e. ,

$$
\left.f(\eta)\right|_{\beta=\Delta \beta}=\left.f(\eta)\right|_{\beta=0}+G(\eta) \Delta \beta .
$$

This process is then repeated to calculate the solutions of equations (6) for $\beta=2 \Delta \beta, \beta=3 \Delta \beta, \ldots$. etc. 


\subsubsection{Solution of Blasius equation by DTM}

To solve (6) using the differential transformation method, it is first necessary to solve the Blasius equation $(\beta=0)$, i.e.,

$$
\frac{d^{3} f(\eta)}{d \eta^{3}}+f(\eta) \frac{d f^{2}(\eta)}{d \eta^{2}}=0
$$

The boundary conditions are given by

$$
\begin{gathered}
f(0)=f^{\prime}(0)=0 \quad \text { at } \quad \eta=0 . \\
f^{\prime}(\infty)=1 \quad \text { as } \quad \eta \rightarrow \infty
\end{gathered}
$$

The boundary value problems ( (29)-(31)) can then be reduced to a pair of initial value problems, which are given by

$$
\frac{d^{3} F(\zeta)}{d \zeta^{3}}+F(\zeta) \frac{d F^{2}(\zeta)}{d \zeta^{2}}=0
$$

The boundary conditions are given by

$$
F(0)=F^{\prime}(0)=0, \quad F^{\prime \prime}(0)=1 \quad \text { at } \quad \zeta=0 .
$$

and by

$$
\frac{d^{3} f(\eta)}{d \eta^{3}}+f(\eta) \frac{d f^{2}(\eta)}{d \eta^{2}}=0
$$

with initial conditions of

$$
f(0)=f^{\prime}(0)=0 \quad f^{\prime \prime}(0)=\left(\frac{1}{d F(\infty) / d \zeta}\right)^{3 / 2}
$$

These equations suggest a transformation of the form

$$
F(\zeta)=\lambda^{-1 / 3} f(\eta), \quad \zeta=\lambda^{1 / 3} \eta, \quad \lambda=\left(\frac{1}{d F(\infty) / d \zeta}\right)
$$

The differential transformation method is then used to solve the pair of initial value problems ((32)-(35)). Initially, the following expressions are defined:

$$
y(\zeta)=\frac{d F(\zeta)}{d \zeta}
$$

and 


$$
z(\zeta)=\frac{d y(\zeta)}{d \zeta}=\frac{d^{2} F(\zeta)}{d \zeta^{2}}
$$

Thereafter, the third-order ordinary differential equation, (32), is reduced to a first-order ordinary differential equation with the following form:

$$
\frac{d z(\zeta)}{d \zeta}+F(\zeta) z(\zeta)=0
$$

The initial conditions become

$$
F(0)=y(0)=0, \quad z(0)=1
$$

By a process of inverse differential transformation, the solutions of each sub-domain take $m+1$ terms for the power series, i.e.,

$$
\begin{array}{ll}
F_{i}(\zeta)=\sum_{k=0}^{m}\left(\frac{\zeta}{H_{i}}\right)^{k} \bar{F}_{i}(k), \quad 0 \leq \zeta \leq H_{i}, \\
y_{i}(\zeta)=\sum_{k=0}^{m}\left(\frac{\zeta}{H_{i}}\right)^{k} Y_{i}(k), \quad 0 \leq \zeta \leq H_{i}, \\
z_{i}(\zeta)=\sum_{k=0}^{m}\left(\frac{\zeta}{H_{i}}\right)^{k} Z_{i}(k), \quad 0 \leq \zeta \leq H_{i},
\end{array}
$$

where $i=0,1,2, \ldots, n$ indicates the $i$-th sub-domain, $k=0,1,2, \ldots, m$ represents the number of terms of the power series, $H_{i}$ represents the sub-domain interval, and $\bar{F}_{i}(k), Y_{i}(k)$ and $Z_{i}(k)$ are the transformed functions of $F_{i}(\zeta)$, $y_{i}(\zeta)$ and $z_{i}(\zeta)$, respectively.

From the initial conditions (40) and the solution equations, (41)-(43), it can be seen that

$$
\begin{gathered}
\bar{F}_{0}(0)=0, \\
Y_{0}(0)=0, \\
Z_{0}(0)=\delta(k)
\end{gathered}
$$

Performing differential transformation of Eqs. (37)(39) gives the following:

$$
\frac{k+1}{H_{i}} \bar{F}_{i}(k+1)=Y_{i}(k),
$$




$$
\begin{gathered}
\frac{k+1}{H_{i}} Y_{i}(k+1)=Z_{i}(k), \\
\frac{k+1}{H_{i}} Z_{i}(k+1)+\sum_{l=0}^{k} \bar{F}_{i}(k-l) Z_{i}(l)=0 .
\end{gathered}
$$

The various values of $\bar{F}_{i}(k), Y_{i}(k)$ and $Z_{i}(k)$ are obtained by using (47)-(49), together with the transformed initial conditions, i.e., (44)-(46). The solution of (32) is then determined by means of the inverse transformed equations, i.e., (41)-(43).

From (42), it can be shown that the value of $d F(\infty) / d \zeta$ approaches a limiting value in the final sub-domain. In the expressions which follow, this limiting value is represented by the parameter " $\lambda$ ". The following expressions are also defined:

$$
u(\eta)=\frac{d f(\eta)}{d \eta}
$$

and

$$
v(\eta)=\frac{d u(\eta)}{d \eta}=\frac{d^{2} f(\eta)}{d \eta^{2}}
$$

Hence, the third-order ordinary differential equation, (34), becomes a firstorder ordinary differential equation with the following form:

$$
\frac{d v(\eta)}{d \eta}+f(\eta) v(\eta)=0
$$

The initial conditions become

$$
\begin{gathered}
f(0)=u(0)=0, \quad v(0)=\lambda^{-3 / 2} . \\
f_{i}(\eta)=\sum_{k=0}^{m}\left(\frac{\eta}{H_{i}}\right)^{k} \bar{f}_{i}(k), \quad 0 \leq \eta \leq H_{i}, \\
u_{i}(\eta)=\sum_{k=0}^{m}\left(\frac{\eta}{H_{i}}\right)^{k} U_{i}(k), \quad 0 \leq \eta \leq H_{i}, \\
v_{i}(\eta)=\sum_{k=0}^{m}\left(\frac{\eta}{H_{i}}\right)^{k} V_{i}(k), \quad 0 \leq \eta \leq H_{i},
\end{gathered}
$$

where as before $i=0,1,2, \ldots, n$ indicates the $i-t h$ sub-domain, $k=$ $0,1,2, \ldots, m$ represents the number of terms of the power series, $H_{i}$ represents 
the sub-domain interval, and $\bar{f}_{i}(k), U_{i}(k)$ and $V_{i}(k)$ are the transformed functions of $f_{i}(\eta), u_{i}(\eta)$ and $v_{i}(\eta)$, respectively.

From the initial conditions (53) and the solution equations, (54)-(56), it can be shown that

$$
\begin{gathered}
\bar{f}_{0}(0)=0, \\
U_{0}(0)=0, \\
V_{0}(0)=\lambda \delta(k)
\end{gathered}
$$

equations (50)-(52) undergo a process of differential transformation to give the following:

$$
\begin{gathered}
\frac{k+1}{H_{i}} \bar{f}_{i}(k+1)=U_{i}(k), \\
\frac{k+1}{H_{i}} U_{i}(k+1)=V_{i}(k), \\
\frac{k+1}{H_{i}} V_{i}(k+1)+\sum_{l=0}^{k} \bar{f}_{i}(k-l) V_{i}(l)=0 .
\end{gathered}
$$

As in the solution of the previous initial value problem, when the various values of $\bar{f}_{i}(k), U_{i}(k)$ and $V_{i}(k)$ have been determined by using (60)-(62), together with the transformed initial conditions ( (57)-(59)), the solution of equations (34) can be obtained by means of the inverse transformed equations, i.e., (54)-(56).

Since the solutions of the boundary value problems $((29)-(31))$ can be established from the previous calculations, $f(\eta)$ is also known and can be substituted into (6) to solve the Falkner-Skan equation.

The differential transformation method is then used to solve the pair of initial value problems given by (23)-(26). Initially, the following expressions are defined:

$$
r(\eta)=\frac{d P(\eta)}{d \eta}
$$

and

$$
s(\eta)=\frac{d r(\eta)}{d \eta}=\frac{d^{2} P(\eta)}{d \eta^{2}}
$$


Thereafter, the third-order ordinary differential equation, (23), is reduced to a first-order ordinary differential equation with the following form:

$$
\frac{d s(\eta)}{d \eta}+f(\eta) s(\eta)+\frac{d^{2} f(\eta)}{d \eta} P(\eta)-\beta r(\eta)=\frac{d f(\eta)}{d \eta}-1
$$

The initial conditions become

$$
P(0)=r(0)=s(0)=0
$$

As before, inverse differential transformation is used to yield the following solutions:

$$
\begin{array}{ll}
P_{i}(\eta)=\sum_{k=0}^{m}\left(\frac{\eta}{H_{i}}\right)^{k} \bar{P}_{i}(k), & 0 \leq \eta \leq H_{i}, \\
r_{i}(\eta)=\sum_{k=0}^{m}\left(\frac{\eta}{H_{i}}\right)^{k} R_{i}(k), & 0 \leq \eta \leq H_{i}, \\
s_{i}(\eta)=\sum_{k=0}^{m}\left(\frac{\eta}{H_{i}}\right)^{k} S_{i}(k), & 0 \leq \eta \leq H_{i},
\end{array}
$$

where $i=0,1,2, \ldots, n$ indicates the $i-t h$ sub-domain, $k=0,1,2, \ldots, m$ represents the number of terms of the power series, $H_{i}$ represents the subdomain interval, and $\bar{P}_{i}(k), R_{i}(k)$ and $S_{i}(k)$ are the transformed functions of $P_{i}(\eta), r_{i}(\eta)$ and $s_{i}(\eta)$, respectively.

From the initial conditions (66) and the solution equations, (67)-(68), it can be shown that

$$
\begin{aligned}
& \bar{P}_{0}(0)=0, \\
& R_{0}(0)=0, \\
& S_{0}(0)=0 .
\end{aligned}
$$

Equations (64)-(65) undergo a process of differential transformation to give the following:

$$
\begin{aligned}
& \frac{k+1}{H_{i}} \bar{P}_{i}(k+1)=R_{i}(k), \\
& \frac{k+1}{H_{i}} R_{i}(k+1)=S_{i}(k),
\end{aligned}
$$


$\frac{k+1}{H_{i}} S_{i}(k+1)+\sum_{l=0}^{k} \bar{f}_{i}(k-l) S_{i}(l)+\sum_{l=0}^{k} \bar{Z}_{i}(k-l) \bar{P}_{i}(l)-\beta R_{i}(k)=\frac{k+1}{H_{i}} f_{i}(k+1)-\delta(k)$.

As in the solution of the previous initial value problem, when the various values of $\bar{P}_{i}(k), R_{i}(k)$ and $S_{i}(k)$ have been determined by using (73)-(75), together with the transformed initial conditions ( (70)-(72)), the solution of equation (65) can be obtained by means of the inverse transformed equations, i.e., (67)-(69).

From $(68$, it is noted that the value of $d P(\infty) / d \eta$ approaches a limiting value in the final sub-domain. The following expression is defined:

$$
A(\eta)=\frac{d Q(\eta)}{d \eta}
$$

and

$$
B(\eta)=\frac{d A(\eta)}{d \eta}=\frac{d^{2} Q(\eta)}{d \eta^{2}}
$$

Thereafter, the third-order ordinary differential equation, (25), is reduced to a first-order ordinary differential equation with the following form:

$$
\frac{d B(\eta)}{d \eta}+f(\eta) B(\eta)+\frac{d^{2} f(\eta)}{d \eta} Q(\eta)-\beta A(\eta)=0
$$

The initial conditions become

$$
Q(0)=A(0)=B(0)=0
$$

The inverse differential transformation is used to yield the following solutions:

$$
\begin{array}{ll}
Q_{i}(\eta)=\sum_{k=0}^{m}\left(\frac{\eta}{H_{i}}\right)^{k} \bar{Q}_{i}(k), & 0 \leq \eta \leq H_{i}, \\
A_{i}(\eta)=\sum_{k=0}^{m}\left(\frac{\eta}{H_{i}}\right)^{k} \bar{A}_{i}(k), & 0 \leq \eta \leq H_{i}, \\
B_{i}(\eta)=\sum_{k=0}^{m}\left(\frac{\eta}{H_{i}}\right)_{i}^{k} \bar{B}_{i}(k), & 0 \leq \eta \leq H_{i},
\end{array}
$$

where $i=0,1,2, \ldots, n$ indicates the $i-t h$ sub-domain, $k=0,1,2, \ldots, m$ represents the number of terms of the power series, $H_{i}$ represents the subdomain interval, and $\bar{Q}_{i}(k), \bar{A}_{i}(k)$ and $\bar{B}_{i}(k)$ are the transformed functions of 
$Q_{i}(\eta), A_{i}(\eta)$ and $B_{i}(\eta)$, respectively.

From the initial conditions (79) and the solution equations, (80)(82), it can be shown that

$$
\begin{aligned}
& \bar{Q}_{0}(0)=0, \\
& \bar{A}_{0}(0)=0, \\
& \bar{B}_{0}(0)=0
\end{aligned}
$$

Equations (76)-(78) undergo a process of differential transformation to give the following:

$$
\begin{gathered}
\frac{k+1}{H_{i}} \bar{Q}_{i}(k+1)=\bar{A}_{i}(k), \\
\frac{k+1}{H_{i}} \bar{A}_{i}(k+1)=\bar{B}_{i}(k), \\
\frac{k+1}{H_{i}} \bar{B}_{i}(k+1)+\sum_{l=0}^{k} \bar{f}_{i}(k-l) \bar{B}_{i}(l)+\sum_{l=0}^{k} \bar{Z}_{i}(k-l) \bar{Q}_{i}(l)-\beta A_{i}(k)=\delta(k) .
\end{gathered}
$$

As in the solution of the previous initial value problem, when the various values of $\bar{Q}_{i}(k), \bar{A}_{i}(k)$ and $\bar{B}_{i}(k)$ have been determined by using (86)-(88), together with the transformed initial conditions ( (83)-(85)), the solution of equation (78) can be obtained by means of the inverse transformed equations, i.e., (80)-(82).

From (81), it is noted that the value of $d Q(\infty) / d \eta$ approaches a limiting value in the final sub-domain.

The value of $C$ is determined by substituting the values of $d P(\infty) / d \eta$ and $d Q(\infty) / d \eta$ into $(27) . C, P(\eta), Q(\eta)$ and their derivatives are then substituted into (22) to determine the value of $G(\eta)$. Finally, $G(\eta)$ is substituted into (28) to generate the solutions of (6) for various values of $\beta$.

\section{Results and discussion}

Equation (6) subject to the the boundary conditions (7) is solved by NIT and $\mathrm{DTM}$, for the pertinent magnetic parameter $\beta$. In order to verify the accuracy of the present methods, the results are compared with those by Cebeci and Keller[5] and Smith [16] for the skin friction coefficient $f^{\prime \prime}(0)$ at $\beta=0$. They are found to be in good agreement ( at $\beta=0$ ). The values of $f^{\prime \prime}(0)$ by DTM and NIT are given in the Table 1 . The values are in close agreement. From the Table 2 and equation (11), it is evident that the values of the skin-friction on the surface of the plate go on increasing as the magnetic parameter $\beta$ goes 
on increasing and physically this appears to be plausible. The skin-friction becomes unity as $\beta \rightarrow \infty$ ( by NIT).

The solutions of the initial value problem (32) and (34) are depicted in Table 2 from which it can be seen that $d F(\infty) / d \zeta$ and $d f(\infty) / d \eta$ approach a limiting value of 1.655190 and 0.999999 respectively. Substituting the value of $d F(\infty) / d \zeta$ into (36) gives a calculated value of $\lambda$ equal to 0.469600 . Using this value of $\lambda$, Table 2 presents the current numerical results for the FalknerSkan boundary-layer problem for the case of $\beta=0$ in terms of $f(\eta)$ and its derivatives. The results obtained by the present method are in good agreement with those provided by White [18] and Kuo [10] to about five decimal places. The variation of the values of $f(\eta)$ and its derivatives for the case of $\beta=0$ are plotted in Fig.1. Figure 2 plots the numerical values of $f(\eta)$ for the present boundary-layer equation it can be seen that as the magnetic parameter increases $f(\eta)$ also increases. Fig. 3 displays the dimensionless velocity profiles $f^{\prime}(\eta)$ for varies magnetic parameter $\beta$. The result show that increasing magnetic parameter is to accelerate the velocity. Fig. 4 show the shear-stress profiles for a flat plate for a wide range of the magnetic parameter. Once again, the current numerical results obtained by NIT and DTM are in good agreement.

Table 1: Comparison of the values of $f^{\prime \prime}(0)$ for for the magnetic parameter $\beta$

\begin{tabular}{lllll}
\hline$\beta$ & Smith [16] & Cebeci [5] & DTM (Present Case) & NIT(Present Case) \\
\hline 0.0 & 0.46960 & 0.46960 & 0.46910 & 0.46920 \\
0.2 & - & - & 0.66343 & 0.64819 \\
0.4 & - & - & 0.80009 & 0.78749 \\
0.6 & - & - & 0.91659 & 0.90562 \\
0.8 & - & - & 1.01988 & 1.01002 \\
1.0 & - & - & 1.11362 & 1.10460 \\
1.2 & - & - & 1.20006 & 1.19170 \\
1.4 & - & - & 1.28068 & 1.27285 \\
1.6 & - & - & 1.35652 & 1.34913 \\
1.8 & - & - & 1.42834 & 1.42132 \\
2.0 & - & - & 1.49671 & 1.49002 \\
$\infty$ & - & - & 1 & - \\
\hline
\end{tabular}

\section{Concluding Remarks}

By NIT, it is important and interesting to note that remarkably simple approximate solutions in closed form as given by (16) - (18) of equation (6) - 


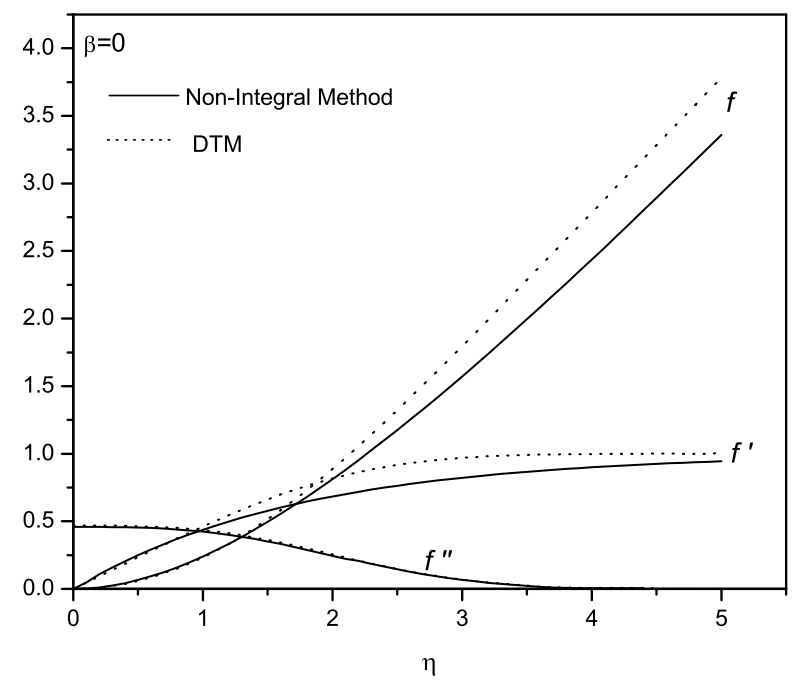

Figure 1: Variation of $f(\eta), f^{\prime}(\eta)$ and $f^{\prime \prime}(\eta)$ for $\beta=0$ by NIT and DTM.

(7), are now available for a wide range of magnetic parameter $\beta$. Also, this method utilizes the 'similarity' between equation (6) and another third order non-linear equation (8), and minimizes the total error. As a matter of this fact, it is hoped that such an analysis will find wider use in engineering applications. By DTM, it has been demonstrated that the current results for the velocity and shear-stress profiles are in good agreement with those provided by NIT. It is authors' belief that the methods presented in this paper provide an effective numerical scheme for determining the solutions of the nonlinear third order differential equations pertaining to the boundary-layer problems.

\section{ACKNOWLEDGEMENTS}

The authors are highly obliged to Professor A. K. Singh of the Department of Mathematics of Banaras Hindu University, Varanasi (U.P), India for his able guidance in the preparation of the manuscript of the paper.

\section{References}

[1] Annamalai, K., Lau, S.C. and Kondepudi, S.N., Non-integral technique for the approximate solution of the transport problems, International Communications in Heat and Mass Transfer 13(1985), 523-534.

[2] Aziz, A. and Na, T. Y., New Approach to the Solution of Falkner-Skan Equation, AIAA Journal, 19(1981), 1242-1244. 


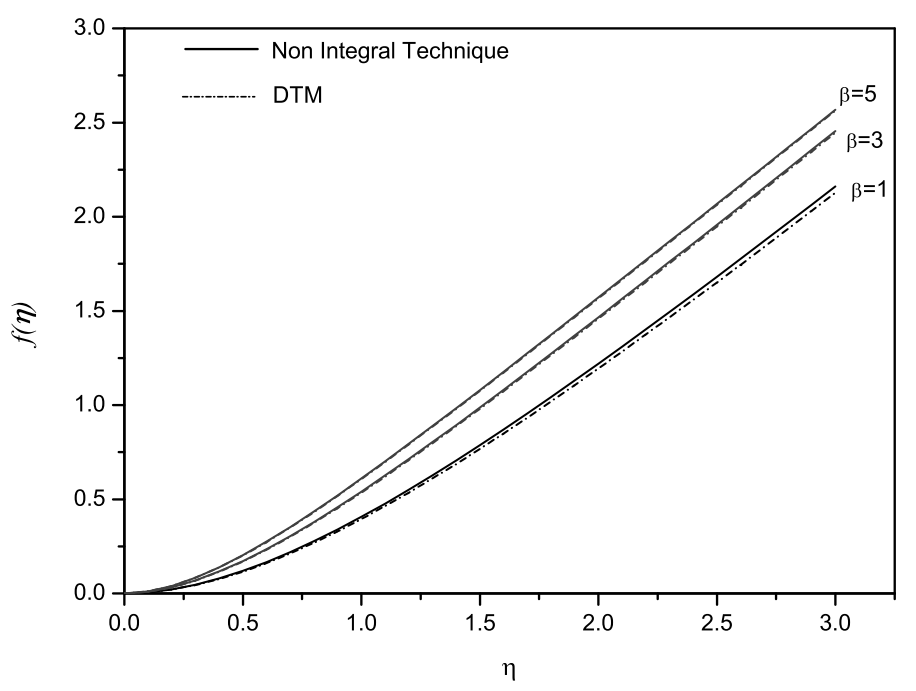

Figure 2: Variation of $f(\eta)$ for $\beta=1,3,5$ by NIT and DTM.

[3] Bairstow, L., Skin friction, J. Roy. Aero. Soc. 19 (1925), 3-12.

[4] Blasius, H., Greinzschichten in fliissigkeiten mit Kleiner reibung, Z. Math. Phys. 56(1908), 1-37.

[5] Cebeci, T. and Keller, H. B., "Shooting and Parallel Shooting Methods for Solving the Falkner-Skan Boundary Layer Equation," Journal of Computational Physics, 7 (1971), 289-300.

[6] Dey, J., A simple technique for approximate solutions of the Falkner-Skan equation, Acta Mechanica 77 (1989), 299-305.

[7] Evans, H.L., Laminar boundary layer theory, Addison - Wesley (1968), $27-28$.

[8] Falkner, V.M. and Skan, S.W., Solution of the boundary layer equations, Phil. Mag. 12(1931), 865-896.

[9] Goldstein, S., Concerning some solutions of the boundary layer equations in hydrodynamics, Proc. Camb. Phil. Soc. 26(1930), 1-30.

[10] Kuo, B.-L., Application of the differential transformation method to the solutions of Falkner-Skan wedge flow, Acta Mechanica 164, 161-174 (2003). 


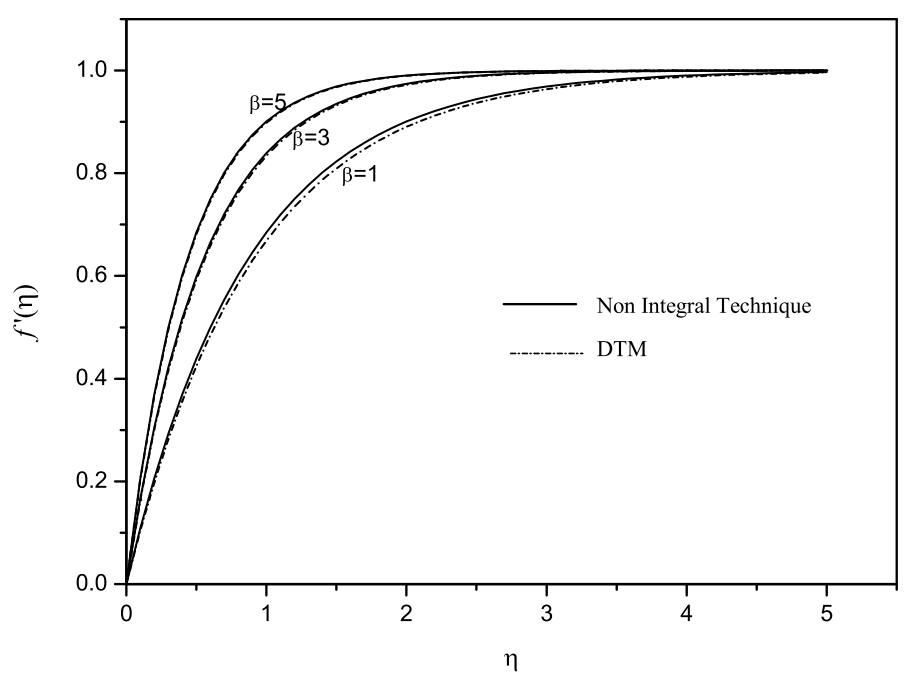

Figure 3: Variation of $f^{\prime}(\eta)$ for $\beta=1,3,5$ by NIT and DTM.

[11] Liao, S. J., A uniformly valid analytic solution of two-dimensional viscous flow over a semi-infinite flat plate. J. Fluid Mech. 385(1999), 101-128.

[12] Pohlhausen, E., Der Warmeaustausch zwischen festen korpern and flussigkeiten mit Kleiner reibung and Kleiner warmeleifung, ZAMM, 1(1921), 115 .

[13] Prandtl, L., The mechanics of viscous fluids, In : W.E. durandi aerodynamic theory III, Springer - Verlag, Berlin (1935), 34-208.

[14] Schlichting, H., Boundary layer theory, McGraw Hill (1st ed.), 1955, 128.

[15] Singh, B.B. and Chandarki, I.M., Existence, uniqueness and asymptotic behaviours of MHD boundary layer flows of an incompressible fluid past a flat plate, Int. J. Math. Sci. \& Engg. Appls. 2(II) (2008), 141-153.

[16] Smith, A.M.O., Improved solutions of Falkner-Skan boundary layer equation, Fund Paper, Journal of the Aerospace Sciences, Sherman M. Fairchild, 1954.

[17] Toepfer, C., Bemerkungen zu dem aufsatz von H. Blasius : Gremzschichten in flissigkeiten mit kleiner reibung, Z. Math. Phys. 60(1912), 397-398.

[18] White, F.M., Viscous fluid flow, 2nd ed. New York: McGraw-Hill 1991. 


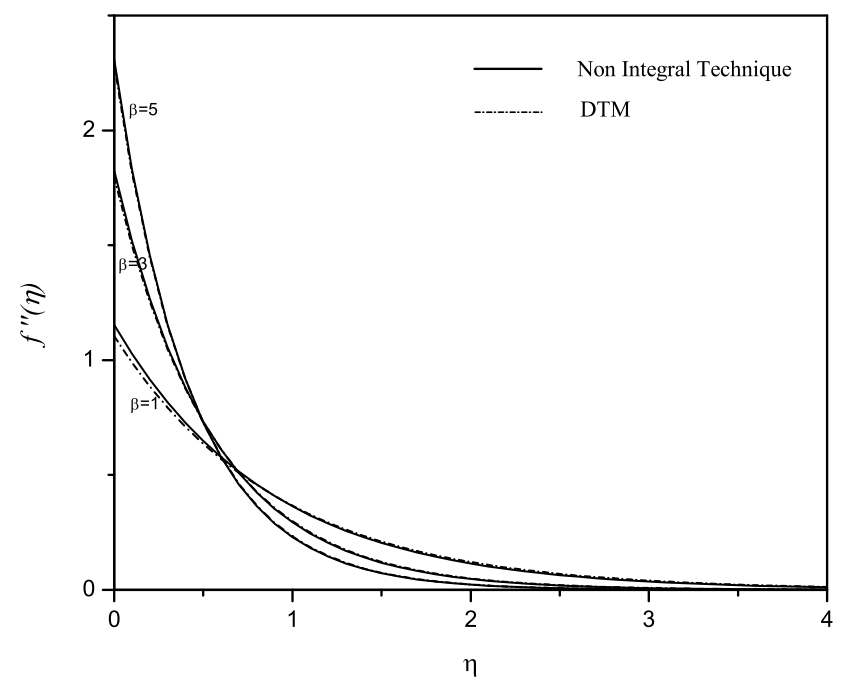

Figure 4: Variation of $f^{\prime \prime}(\eta)$ for $\beta=1,3,5$ by NIT and DTM.

[19] Yu, L. T. and Chen, C.K., The solution of the Blasius equation by the differential transformation method. Mathl. Comp. Model. 28(1998), 101111.

[20] Zhou, X., Differential transformation and its applications for electrical circuits (in Chinese). Wuhan, China: Huazhong University Press 1986. 
Table 2: Values of $F(\zeta), f(\eta)$ and their derivatives from the initial value problems (32) - (33) and (34) - (35)

\begin{tabular}{lllllll}
\hline$\eta, \zeta$ & $F(\zeta)$ & $F^{\prime}(\zeta)$ & $F^{\prime \prime}(\zeta)$ & $f(\eta)$ & $f^{\prime}(\eta)$ & $f^{\prime \prime}(\eta)$ \\
\hline 0.00 & 0.000000 & 0.000000 & 1.000000 & 0.000000 & 0.000000 & 0.469600 \\
0.20 & 0.019997 & 0.199933 & 0.998668 & 0.009391 & 0.093905 & 0.469306 \\
0.40 & 0.079915 & 0.398937 & 0.989396 & 0.037549 & 0.187605 & 0.467254 \\
0.60 & 0.179357 & 0.594661 & 0.964703 & 0.084386 & 0.280575 & 0.461734 \\
0.80 & 0.317314 & 0.783380 & 0.918537 & 0.149674 & 0.371963 & 0.451190 \\
1.00 & 0.491930 & 0.960417 & 0.847633 & 0.232990 & 0.460633 & 0.434379 \\
1.20 & 0.700371 & 1.120825 & 0.752765 & 0.333657 & 0.545246 & 0.410565 \\
1.40 & 0.938860 & 1.260271 & 0.639249 & 0.450724 & 0.624386 & 0.379692 \\
1.60 & 1.202886 & 1.375886 & 0.516206 & 0.582956 & 0.696700 & 0.342487 \\
1.80 & 1.487566 & 1.466848 & 0.394557 & 0.728872 & 0.761057 & 0.300445 \\
2.00 & 1.788066 & 1.534483 & 0.284413 & 0.886797 & 0.816695 & 0.255669 \\
2.20 & 2.100006 & 1.581860 & 0.192824 & 1.054947 & 0.863304 & 0.210580 \\
2.40 & 2.419731 & 1.613053 & 0.122720 & 1.231528 & 0.901065 & 0.167560 \\
2.60 & 2.744434 & 1.632327 & 0.073226 & 1.414824 & 0.930601 & 0.128613 \\
2.80 & 3.072123 & 1.643492 & 0.040933 & 1.603284 & 0.952875 & 0.095113 \\
3.00 & 3.401492 & 1.649552 & 0.021426 & 1.795568 & 0.969055 & 0.067710 \\
3.20 & 3.731747 & 1.652633 & 0.010499 & 1.990581 & 0.980365 & 0.046370 \\
3.40 & 4.062439 & 1.654099 & 0.004816 & 2.187467 & 0.987970 & 0.030535 \\
3.60 & 4.393333 & 1.654753 & 0.002067 & 2.385590 & 0.992888 & 0.019329 \\
3.80 & 4.724315 & 1.655025 & 0.000831 & 2.584499 & 0.995944 & 0.011759 \\
4.00 & 5.055332 & 1.655132 & 0.000312 & 2.783886 & 0.997770 & 0.006874 \\
4.20 & 5.386363 & 1.655171 & 0.000110 & 2.983555 & 0.998818 & 0.003861 \\
4.40 & 5.717399 & 1.655184 & 0.000036 & 3.183383 & 0.999397 & 0.002084 \\
4.60 & 6.048437 & 1.655189 & 0.000011 & 3.383296 & 0.999703 & 0.001081 \\
4.80 & 6.379474 & 1.655190 & 0.000003 & 3.583254 & 0.999859 & 0.000538 \\
5.00 & 6.710512 & 1.655190 & 0.000001 & 3.783235 & 0.999936 & 0.000258 \\
\hline & & & & & &
\end{tabular}

